\title{
ANTIBIOTIC RESISTANCE PATTERNS OF URINARY TRACT INFECTIONS IN A NORTHEASTERN BRAZILIAN CAPITAL
}

\author{
Mirella Alves CUNHA, Gabriela Lins Medeiros ASSUNÇÃo, Iara Marques MEDEIROS \& Marise Reis FREITAS
}

\begin{abstract}
SUMMARY
Urinary tract infection is a common problem worldwide. Its clinical characteristics and susceptibility rates of bacteria are important in determining the treatment of choice and its duration. This study assessed the frequency and susceptibility to antimicrobials of uropathogens isolated from community-acquired urinary tract infections in the city of Natal, Rio Grande do Norte State capital, northeastern Brazil, from 2007 to 2010. A total of 1,082 positive samples were evaluated; E. coli was the most prevalent pathogen $(60.4 \%)$. With respect to the uropathogens susceptibility rates, the resistance of enterobacteria to ciprofloxacin and sulfamethoxazoletrimethoprim was $24.4 \%$ and $50.6 \%$, respectively. Susceptibility was over $90 \%$ for nitrofurantoin, aminoglycosides and third-generation cephalosporins. High resistance rates of uropathogens to quinolones and sulfamethoxazole-trimethoprim draws attention to the choice of these drugs on empirical treatments, especially in patients with pyelonephritis. Given the increased resistance of community bacteria to antimicrobials, local knowledge of susceptibility rates of uropathogens is essential for therapeutic decision making regarding patients with urinary tract infections.
\end{abstract}

KEYWORDS: Urinary tract infections; Antibacterial drug resistance; Enterobacteriaceae; Antibacterial agents.

\section{INTRODUCTION}

Urinary tract infections (UTI) are frequent problems, exceeded only by respiratory and gastrointestinal infections. It affects around 150 million people worldwide each year ${ }^{1,2}$. UTI is defined as a tissue invasion by means of bacterial colonization of any part of the urinary tract, from the urethra to the kidneys ${ }^{3}$. Thus, it may involve the lower or upper urinary tract. Episodes of UTI occurring in premenopausal, nonpregnant, women with no known urological abnormalities are classified as uncomplicated, while all others are considered complicated UTI. This concept and knowledge of local susceptibility rates are important in decision making of the optimal choice for empirical antimicrobial therapy and its duration ${ }^{1,2}$.

Most UTIs are caused by Gram-negative bacteria, being Escherichia coli the most commonly isolated. Other bacteria involved are Proteus mirabilis, Klebsiella pneumoniae, Pseudomonas aeruginosa, Enterococcus spp., Enterobacter spp., Group B Streptococcus and Staphylococcus saprophyticus ${ }^{3-5}$. Variations in microbial spectrum and susceptibility patterns may occur in different regions, and the previous use of antimicrobials is an important predictor of resistance ${ }^{5,6}$.

The high incidence of these infections and the need to start treatment before the results of microbiological findings become available often lead to the adoption of empirical therapy ${ }^{5}$. National and international studies have shown increased resistance to commonly used antimicrobials ${ }^{7-9}$. Besides, knowledge of the resistance pattern of uropathogens is important in guiding optimal antimicrobial choice in the initial approach of the patient. The aim of this study was to describe pathogens frequency and their susceptibility patterns in community-acquired urinary tract infections in the city of Natal, Rio Grande do Norte State capital, northeastern Brazil.

\section{MATERIALS AND METHODS}

This is a retrospective study to evaluate the results of urine cultures taken at the Central State Laboratory (LACEN/RN), a reference center for microbiological examinations in the public health system of Rio Grande do Norte State, Brazil. Data were based on records collected from January 2007 to December 2010, precluding identification of the subjects involved.

The urine specimens were processed and incubated on CLED agar, at a temperature of $35 \pm 2{ }^{\circ} \mathrm{C}$, for $24-48$ hours. A count $\geq 100,000$ colony forming units (CFU) per milliliter of urine was considered positive after incubation, and these isolates were identified. The antimicrobial susceptibility was determined on urinary isolates using the disk diffusion method, according to CLSI (Clinical \& Laboratory Standard Institute)

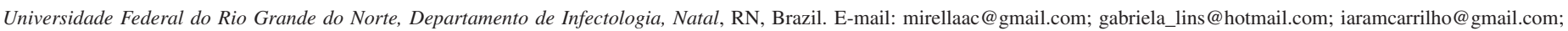
mariserf@gmail.com

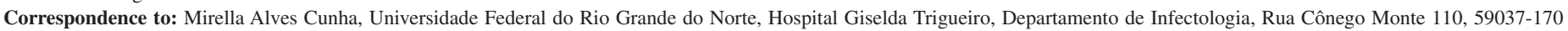
Natal, RN, Brasil. E-mail: mirellaac@gmail.com 


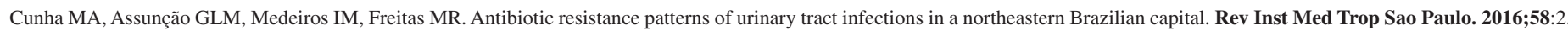

Table 1

Antimicrobial susceptibility of the three main uropathogens from Family Enterobacteriaceae . January 2007 to December 2010 , LACEN/RN.

\begin{tabular}{lccc}
\hline & & \% Susceptible & \\
\cline { 2 - 4 } Antimicrobials & $\begin{array}{c}\text { E.coli } \\
(\mathrm{n}=653)\end{array}$ & $\begin{array}{c}\text { Klebsiella spp. } \\
(\mathrm{n}=154)\end{array}$ & $\begin{array}{c}\text { Proteus spp. } \\
(\mathrm{n}=52)\end{array}$ \\
\hline Amikacin & 94.1 & 92.7 & 87.5 \\
Gentamicin & 92.1 & 81.7 & 78.1 \\
Ampicillin & 48.3 & - & - \\
Ampicillin/sulbactam & 52.0 & 72.2 & 80.0 \\
Amoxicillin/Clavulanate & 82.2 & 81.1 & 95.7 \\
Cephalothin & 42.8 & 72.3 & 55.6 \\
Ceftriaxone & 92.2 & 86.1 & 80.0 \\
Cefepime & 90.8 & 73.2 & 76.0 \\
Ciprofloxacin & 75.6 & 75.4 & 69.8 \\
Norfloxacin & 79.6 & 76.5 & 75.0 \\
Nitrofurantoin & 93.4 & 45.1 & 15.6 \\
Sulfamethoxazole/Trimethoprim & 49.4 & 65.6 & 46.2 \\
Nalidixic Acid & 68.4 & 60.6 & 44.4 \\
\hline
\end{tabular}

recommendations. In addition, susceptibility test results were interpreted following CLSI standards and recommendations $(2005)^{10}$ and, for the purpose of the analysis, intermediate susceptibility was interpreted as resistance. Only aerobic bacterial infections were analyzed in this study. Cultures showing fungal growth, mixed growth and those not conclusive in the laboratory, irrespective of reason, were excluded.

The data were analyzed using Excel 2007®. Susceptibility to antimicrobials was calculated by species.

\section{RESULTS}

A total of 1,082 samples were considered positive, according to inclusion microbiological criteria. Of these, 836 (79\%) were from women. The median age of patients at time of treatment was 68.3 years (range three months-95 years). Among those with positive samples, 346 (31.9\%) were older than the age of 60; of these, 221 (63.9\% of people aged 60 and over) were female. We do not have data on how many samples were from patients treated in primary care facilities, but, in Natal/RN, the LACEN/RN laboratory is responsible for the execution of exams sent from the primary care facilities, while nosocomial samples are analyzed in hospital laboratories.

Among the positive samples, the most common pathogen isolated was E. coli (653/60.4\%), followed by Klebsiella spp. (154/14.2\%), Staphylococcus spp. (78/7.2\%), and Proteus spp. (52/4.8\%). Data are summarized in Tables 1 and 2.

Resistance to ciprofloxacin was identified in $24.4 \%$ of E. coli and Klebsiella spp., $30.2 \%$ of Proteus spp., and $9.4 \%$ of Staphylococcus spp. Resistance to sulfamethoxazole-trimethoprim was $50.6 \%$ for $E$. coli, $34.4 \%$ for Klebsiella spp., $53.8 \%$ for Proteus spp., and $18.6 \%$

\section{Table 2}

Antimicrobial susceptibility of Staphylococcus spp.isolated from urine. January 2007 to December 2010, LACEN/RN.

\begin{tabular}{lc}
\hline & \% Susceptible \\
\cline { 2 - 2 } Antimicrobials & $\begin{array}{c}\text { Staphylococcus } \mathrm{spp} \\
(\mathrm{n}=78)\end{array}$ \\
\hline Amikacin & 33.3 \\
Gentamicin & 84.3 \\
Ciprofloxacin & 90.6 \\
Norfloxacin & 94.4 \\
Nitrofurantoin & 98.2 \\
Oxacillin & 56.3 \\
Sulfamethoxazole/Trimethoprim & 81.4 \\
Nalidixic Acid & 14.3 \\
\hline
\end{tabular}

for Staphylococcus spp. Susceptibility to ceftriaxone was $92.2 \%$ for E. coli, $86.1 \%$ for Klebsiella spp., and $80.0 \%$ for Proteus spp. E. coli was susceptible to nitrofurantoin in $93.4 \%$ of the samples. Resistance to cephalothin was $57.2 \%$ for E. coli, $27.7 \%$ for Klebsiella spp., and 44.4\% for Proteus spp.; $43.7 \%$ of Staphylococcus spp. were resistant to oxacillin. In relation to aminoglycosides, susceptibility to amikacin was $94.1 \%$ for E. coli, $92.7 \%$ for Klebsiella spp., and $87.5 \%$ for Proteus spp.

\section{DISCUSSION}

The present study provides information regarding species that cause community-acquired urinary tract infection treated at public 
health services in Natal, Brazil, and their susceptibility to the main antimicrobials. Most of the samples were from patients treated at primary care facilities, and $79 \%$ were from women. There was no discrimination between asymptomatic bacteriuria, complicated, and uncomplicated UTI. E. coli predominated among the isolated agents, accounting for $60 \%$ of the positive cultures. This value is lower than that found in a number of international studies and similar to that obtained in Brazilian studies $^{1,5,8,9,11}$, which might reveal a local characteristic, reaffirming the need to know the regional profile in order to choose the correct antibiotic and course of empirical treatment.

Furthermore, we had a high percentage of samples from people aged 60 and over $(31.9 \%)$, predominantly women. Older people, especially women, have the highest incidence of asymptomatic bacteriuria and UTIs and more associated comorbidities, like diabetes, considered an important risk factor for recurrent UTIs in women ${ }^{12}$. The antimicrobial use in this group is very frequent, because of recurrent UTIs, mistaken treatment for asymptomatic bacteriuria, or treatment of others infections ${ }^{12}$. It is possible that part of the study population had history of previous antimicrobial use or previous hospitalization. These factors have probably contributed to pathogen distribution and the sensitivity profile in this study, with high resistance of Gram-negative Enterobacteriaceae to quinolones and high resistance of Staphylococcus spp. to oxacillin.

Escherichia coli, Klebsiella spp., and Staphylococcus spp. were isolated in $82 \%$ of the samples, and most were susceptible to nitrofurantoin (>92\%), excepting for Klebsiella spp. strains (45\%). Therefore, these data reinforce the recommendation of various clinical guidelines related to the empirical prescription of nitrofurantoin for uncomplicated cystitis. Fosfomycin, also recommended for these situations, was not tested in our study ${ }^{7,13-15}$.

Fluoroquinolones are widely used for empirical treatment of UTI, including the cases of upper urinary tract infections ${ }^{9}$. We observed high resistance rates of the main isolated species to the tested antimicrobials (> 25\% for E. coli). Similar data were described by ANDRADE et al., in samples of five Latin American countries (Argentina, Chile, Brazil, Mexico, and Venezuela) ${ }^{7}$. The previous use of quinolones is an independent risk factor for resistance to ciprofloxacin ${ }^{16}$. The indiscriminate use of this class of antimicrobials in community-acquired infections may have contributed to the high percentage of resistance observed, considering that the Brazilian population had free over-thecounter access to antimicrobials until 2011 (Resolution No. 20 of the Collegiate Board - RDC, on May 5, 2011). Therefore, quinolones as empirical therapy must be considered carefully, especially for patients with pyelonephritis. In this respect, if susceptibility is confirmed by pathogen isolation, quinolones are alternatives for de-escalation therapy, given the possibility of their oral administration ${ }^{17}$.

Most of the E. coli strains isolated (50.6\%) and other species were resistant to sulfamethoxazole-trimethoprim, as described in earlier studies ${ }^{5,7}$. This finding is most probably due to the wide use of these antimicrobials in the treatment of community-acquired infections. Even though sulfamethoxazole-trimethoprim is one of the alternatives for the empirical treatment of uncomplicated cystitis, therefore this association is not a safe choice. Guidelines of the American Infectious Diseases Society and the European Society for Microbiology and Infectious Diseases suggest that antimicrobials with a resistance rate above $20 \%$ should not be prescribed empirically to patients with uncomplicated cystitis, unless susceptibility is determined by prior isolation in culture ${ }^{17}$.

In pregnant women, asymptomatic bacteriuria and UTI are common problems that should be treated with proper antimicrobials, due to a greater risk of pyelonephritis and the relationship between asymptomatic bacteriuria and obstetric complications ${ }^{18,19}$. The high percentage of strains resistant to first-generation cephalosporins and amoxicillin in our sample limits the empirical use of these drugs in pregnant women. Under these circumstances, nitrofurantoin, fosfomycin or amoxicillin/clavulanate are safer alternatives for cystitis, while third-generation cephalosporins should be used for pyelonephritis, until the susceptibility test of the isolated bacteria is available ${ }^{17,20}$.

The high susceptibility to third-generation cephalosporins and aminoglycosides (>90\% in most strains), as well as their pharmacokinetic characteristics, make them the first line of therapy for pyelonephritis, until microbiological data are known.

The authors underscore the importance of knowledge of the local antimicrobial susceptibility pattern in the decision making of the therapy for UTI, given the increased resistance of community bacteria to antimicrobials commonly prescribed for this condition.

\section{REFERENCES}

1. Hooton TM. Clinical practice. Uncomplicated urinary tract infection. N Engl J Med 2012;366:1028-37.

2. Hsueh PR, Hoban DJ, Carmeli Y, Chen SY, Desikan S, Alejandria M, et al. Consensus review of the epidemiology and appropriate antimicrobial therapy of complicated urinary tract infections in Asia-Pacific region. J Infect. 2011;63:114-23.

3. Saadeh SA, Mattoo TK. Managing urinary tract infections. Pediatr Nephrol. 2011;26:196776.

4. Akram M, Shahid M, Khan AU. Etiology and antibiotic resistance patterns of communityacquired urinary tract infections in J N M C Hospital Aligarh, India. Ann Clin Microbiol Antimicrob. 2007;6:4.

5. Braoios A, Turatti TF, Meredija LCS, Campos TRS, Denadai FHM. Infecções do trato urinário em pacientes não hospitalizados: etiologia e padrão de resistência aos antimicrobianos. J Bras Patol Med Lab. 2009;45:449-56.

6. Kiffer CR, Camargo EC, Shimakura SE, Ribeiro PJ Jr, Bailey TC, Pignatari AC, et al. A spatial approach for the epidemiology of antibiotic use and resistance in communitybased studies: the emergence of urban clusters of Escherichia coli quinolone resistance in Sao Paulo, Brasil. Int J Health Geogr. 2011;10:17.

7. Andrade SS, Sader HS, Jones RN, Pereira AS, Pignatari AC, Gales AC. Increased resistance to first-line agents among bacterial pathogens isolated from urinary tract infections in Latin America: time for local guidelines?. Mem Inst Oswaldo Cruz. 2006;101:741-8.

8. Kiffer CR, Mendes C, Oplustil CP, Sampaio JL. Antibiotic resistance and trend of urinary pathogens in general outpatients from a major urban city. Int Braz J Urol. 2007;33:428 .

9. Rocha JL, Tuon FF, Johnson JR. Sex, drugs, bugs, and age: rational selection of empirical therapy for outpatient urinary tract infection in an era of extensive antimicrobial resistance. Braz J Infect Dis. 2012;16:115-21. 
10. Clinical and Laboratory Standards Institute. Performance standards for antimicrobial susceptibility testing: fifteenth informational supplement. Wayne: Clinical and Laboratory Standards Institute/NCCLS; 2005.

11. de Lucas Collantes C, Cela Alvargonzalez J, Angulo Chacon AM, Garcia Ascaso M, Pineiro Perez R, Cilleruelo Ortega MJ, et al. Infecciones del tracto urinario: sensibilidad antimicrobiana y seguimiento clínico. An Pediatr(Barc). 2012;76:224-8.

12. Mody L, Juthani-Mehta M. Urinary tract infections in older women: a clinical review. Jama. 2014;311:844-54

13. Kashanian J, Hakimian P, Blute M Jr, Wong J, Khanna H, Wise G, et al. Nitrofurantoin: the return of an old friend in the wake of growing resistance. BJU Int. 2008;102:1634-7.

14. Michalopoulos AS, Livaditis IG, Gougoutas V. The revival of fosfomycin. Int J Infect Dis. 2011;15:e732-9.

15. Raz R. Fosfomycin: an old--new antibiotic. Clin Microbiol Infect. 2012;18:4-7.

16. Killgore KM, March KL, Guglielmo BJ. Risk factors for community-acquired ciprofloxacin-resistant Escherichia coli urinary tract infection. Ann Pharmacother. 2004;38:1148-52
17. Gupta K, Hooton TM, Naber KG, Wullt B, Colgan R, Miller LG, et al. International clinical practice guidelines for the treatment of acute uncomplicated cystitis and pyelonephritis in women: a 2010 update by the Infectious Diseases Society of America and the European Society for Microbiology and Infectious Diseases. Clin Infect Dis 2011;52:e103-20.

18. Mittendorf R, Williams MA, Kass EH. Prevention of preterm delivery and low birth weight associated with asymptomatic bacteriuria. Clin Infect Dis. 1992;14:927-32.

19. Smaill FM, Vazquez JC. Antibiotics for asymptomatic bacteriuria in pregnancy. Cochrane Database Syst Rev. 2015;8:Cd000490.

20. Vazquez JC, Abalos E. Treatments for symptomatic urinary tract infections during pregnancy. Cochrane Database Syst Rev. 2011;19:Cd002256.

Received: 30 July 2014

Accepted: 08 April 2015 